\author{
Principal Investigator: Jan Olek, Purdue University, olek@purdue.edu, 765.494.5015 \\ Program Office: jtrp@purdue.edu, 765.494.6508,www.purdue.edu/jtrp \\ Sponsor: Indiana Department of Transportation, 765.463.1521
}

SPR-3021

2012

\title{
PCC Properties to Support W/C Determination for Durability
}

\section{Introduction}

Concrete, as one of the most widely used construction materials, is very durable and can provide long service life without extensive maintenance. The strength and durability of concrete are primarily a function of its water-cementitious material ratio value $(\mathrm{w} / \mathrm{c})$. This ratio is the mass of water divided by the total mass of cementitious material (sum of the masses of Portland cement (or blended cement) and any additional pozzolanic material such as fly ash, slag, silica fume or natural pozzolans).

Although it is a common practice to account for absorption and actual moisture content of aggregates (as well as for the amount of water added to the batch) when reporting the w/c value of fresh concrete during the trial batches, this information is often not tracked during the actual production of field concrete. As a result, the possibility will always exist that the actual $\mathrm{w} / \mathrm{c}$ of the field mixture will be different from the design (target) w/c value. This difference can occur for three reasons. First, additional water may be purposely added to the mixture prior to the concreting operation to increase the ease of placement and finishing. Second, the water-cement ratio can also change due to the use of aggregates that have absorption values that do not match those used to develop the proportions of the basic mix (the use of aggregates that have lower absorption values will result in a higher w/c value in the batch and vice versa). Finally, the differences in w/c can arise from variability in moisture content of aggregates in the stockpiles.

The use of w/c lower than that specified in the mix design will result in stronger but more brittle concrete, which may also be difficult to place and finish. Similarly, the use of w/c higher than specified will result in concrete that is less strong and less durable. The reason that $w / c$ has such a strong influence on concrete's strength and durability is directly linked to the fact that its value strongly influences the volume and the characteristics of capillary porosity, both of which directly control strength and durability. Since w/c plays such a crucial role in controlling concrete quality, there has always been a need for a tool or procedure that can verify the actual $\mathrm{w} / \mathrm{c}$ value of concrete immediately prior to placement.
Nowadays, there is no standardized technique for determining w/c in fresh concrete. The three standard test procedures that have been historically used to obtain either water and/or cement content of fresh concrete (both of which are needed for w/c calculations) include the following: ASTM C 1078 (standard test method for determining the cement content of freshly mixed concrete), ASTM C 1079 (standard test method for determining the water content of freshly mixed concrete), and AASHTO T 318 (standard test method for water content of freshly mixed concrete using microwave oven drying). Since both of the ASTM standards (C 1078 and C 1079) have been discontinued since 1998 , the only standard currently available for determining water content in fresh concrete is the AASHTO T 318 (microwave oven) method. Since the modern ready mix plants can typically accurately control the amount of cement in the batch, the knowledge of microwave oven determined water content will allow (after being corrected for the amount of water absorbed by the aggregates) for calculation of w/c.

However, the use of microwave oven measured water content for determining $\mathrm{w} / \mathrm{c}$ requires the performance of the additional test. Therefore, the focus of the present study was to explore the feasibility of using unit weight (which is already commonly measured as a part of the quality assurance (QA) programs) to determine w/c values of field concretes.

\section{Findings}

During the course of the present study, it was found that the unit weight of concrete can be used as a tool for determining w/c by following these three steps:

1. Establish the unit weight-w/c relationship. This was done by changing the amount of water in the basic mix (CMD) composition while keeping the value of air content constant. Consequently, the CMD composition as well as the w/c and unit weight will be altered (i.e., new mixture designs will be created). The unit weights of these new mixture designs, along with their w/c values, were correlated using linear regression analysis. 
2. Adjust the measured unit weight. In order to apply the developed unit weight-w/c relationship to determine the w/c values of concrete based on its measured unit weight, corrections may be needed to account for the fact that actual values of air content in the mix and specific gravities of aggregates used in the batch may be different from those used in establishing the w/c-unit weight relationship. The adjustment for the differences in air content specific gravities of aggregates can be performed using equations that have been developed in this study.

3. Determine the actual $w / c$. This was done by using the adjusted measured unit weight as an input into the previously developed unit weight-w/c relationship.

The proposed method of using the unit weight of concrete for determining w/c has been evaluated on both laboratory and field concretes.

The unit weights for laboratory concretes required for this method have been determined either by using a "zero-air" procedure (ZAP) developed as a part of this study or by using conventional (following AASHTO specifications) methods. The ZAP technique was used to verify the w/c of 58 different laboratory concrete mixes. These verification efforts revealed that the minimum, maximum, standard error, and $95^{\text {th }}$ percentile of the differences $(\Delta \mathrm{w} / \mathrm{c})$ between batched and determined w/c were, respectively, $0.000,0.042,0.017$, and 0.030 . The AASHTO determined unit weight (which also required measurements of the actual air content of concrete) was used to verify the w/c values of an additional set of 57 laboratory mixes. When using the AASHTO unit weights (and air contents) the minimum, maximum, standard error, and $95^{\text {th }}$ percentile of $\Delta \mathrm{w} / \mathrm{c}$ of were, respectively, $0.000,0.075,0.030$, and 0.054 .

In the part of the evaluation on field concretes, the AASHTO measured unit weights ware used to determine the $w / c$ values of 22 different field mixtures. For this case, the differences $(\Delta \mathrm{w} / \mathrm{c})$ between the design and unit weightcalculated values of $w / c$ were in the range \pm 0.030 for all but one mixture.
The direct comparison of the results from the proposed unit weight method with the results obtained from the microwave oven method for determining w/c revealed that the former is faster but less accurate. Specifically, when used on five separate concrete samples, the accuracy of the microwave oven method was 0.010 , much smaller than the previously mentioned values of 0.030 for the ZAP and 0.054 for the AASHTO unit weight methods.

\section{Implementation}

The method of using the unit weight of concrete for the determination of $\mathrm{w} / \mathrm{c}$ developed in this study provides a fast and inexpensive tool for quality control. Through the course of laboratory work, the accuracies of this method were found to be 0.030 and 0.054 when were applied to ZAP measured unit weights of non-air-entrained plain concretes and AASHTO measured unit weights of air-entrained ternary concretes.

It is recommended that the implementation part of this study involve further verification of the proposed approach using trial batches because for these batches, the target $\mathrm{w} / \mathrm{c}$ values, along with the moisture content and specific gravities of aggregates, can be well controlled.

\section{Recommended Citation}

Yohannes, Y., and J. Olek. PCC Properties to Support W/C Determination for Durability. Publication FHWA/IN/JTRP2012/24. Joint Transportation Research Program, Indiana Department of Transportation and Purdue University, West Lafayette, Indiana, 2012. doi: 10.5703/1288284314986.

View the full text of this technical report here: http://dx.doi.org/10.5703/1288284314986

Published reports of the Joint Transportation Research Program are available at: http://docs.lib.purdue.edu/jtrp/

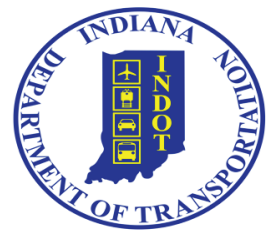

